\title{
Comparative genomics reveals Lysinibacillus sphaericus group comprises a novel species
}

\section{(1) ${ }^{\text {crosemant }}$}

Camilo Gómez-Garzón, Alejandra Hernández-Santana and Jenny Dussán*

\begin{abstract}
Background: Early in the 1990s, it was recognized that Lysinibacillus sphaericus, one of the most popular and effective entomopathogenic bacteria, was a highly heterogeneous group. Many authors have even proposed it comprises more than one species, but the lack of phenotypic traits that guarantee an accurate differentiation has not allowed this issue to be clarified. Now that genomic technologies are rapidly advancing, it is possible to address the problem from a whole genome perspective, getting insights into the phylogeny, evolutive history and biology itself.

Results: The genome of the Colombian strain L. sphaericus OT4b.49 was sequenced, assembled and annotated, obtaining 3 chromosomal contigs and no evidence of plasmids. Using these sequences and the 13 other $L$. sphaericus genomes available on the NCBI database, we carried out comparative genomic analyses that included whole genome alignments, searching for mobile elements, phylogenomic metrics (TETRA, ANI and in-silico DDH) and pan-genome assessments. The results support the hypothesis about this species as a very heterogeneous group. The entomopathogenic lineage is actually a single and independent species with 3728 core genes and 2153 accessory genes, whereas each non-toxic strain seems to be a separate species, though without a clear circumscription. Toxin-encoding genes, bin $A, B$ and $m t \times 1,2,3$ could be acquired via horizontal gene transfer in a single evolutionary event. The non-toxic strain OT4b.31 is the most related with the type strain KCTC 3346.

Conclusions: The current L. sphaericus is actually a sensu lato due to a sub-estimation of diversity accrued using traditional non-genomics based classification strategies. The toxic lineage is the most studied with regards to its larvicidal activity, which is a greatly conserved trait among these strains and thus, their differentiating feature. Further studies are needed in order to establish a univocal classification of the non-toxic strains that, according to our results, seem to be a paraphyletic group.
\end{abstract}

Keywords: Lysinibacillus sphaericus, Pan-genome, Core-genome, Phylogeny, Larvicidal

\section{Background}

Since the discovery of entomopathogenic activity in Bacillus thuringiensis in the 1960s, many bacteria with insecticidal activity have been described. Isolates of $B$. thuringiensis and Lysinibacillus sphaericus are frequently reported [1]. The latter is more active against Culex and Anopheles spp. and more persistent in polluted aquatic environments than $B$. thuringiensis var. israelensis [2, 3]. Lysinibacillus sphaericus is a gram-positive and sporeforming bacteria isolated for the first time from fourthinstar larvae of Culiseta incidens near Fresno, California [4]. However, this strain displayed a low level of toxicity

\footnotetext{
* Correspondence: jdussan@uniandes.edu.co

Centro de Investigaciones Microbiológicas (CIMIC), Universidad de los Andes, Cra 1 N. 18 A-12, Bogotá, Colombia
}

[5] and it was not until the 1970s that the first strains with potential use as mosquito-control agents were discovered [6].

In spite of being widely used in biological control programs, not all strains of $L$. sphaericus are toxic against mosquitoes. Nowadays, it is well known that a plethora of insecticidal toxins are responsible for the entomopathogenic activity of the toxic strains. Binary prototoxin (Bin) is the major insecticidal protein produced by $L$. sphaericus; it is contained inside the parasporal crystal and comprises two proteins: BinA $(42 \mathrm{kDa})$ and $\mathrm{Bin} B$ $(51 \mathrm{kDa})$. After being ingested by larva, these proteins are solubilized in the gut and undergo proteolysis to active lower molecular weight derivatives $[2,7,8]$. Other crystal proteins, Cry 48 and Cry49, might be produced 
on sporulation by some toxic strains. These toxins are related to Cry toxins of B. thuringiensis and Bin family toxins, respectively [1]. L. sphaericus may also produce insecticidal toxins during vegetative stage; this is the case of Mtx proteins [9, 10] whose mode of action remains to be elucidated.

Formerly known as Bacillus sphaericus, L. sphaericus is characterized by having a spherical terminal spore and by its inability to utilize carbohydrates, except $\mathrm{N}$ acetylglucosamine [11]. Instead, it uses organic and amino acids as carbon sources [5]. This species may be found in soil and aquatic environments and, recently, has gained attention because it has shown outstanding potential for environmental and industrial applications beyond biological control, especially in bioremediation of toxic metals [12-14], phosphorous solubilization [15], among others [16].

In 2007, this species was reclassified to a new genus according to phenotypic traits, mainly based on differences in peptidoglycan composition which includes lysine and aspartic acid instead of meso-diaminopimelic acid, the major component of Bacillus cell wall [17]. No genomic support to assess this classification was reported until a few years ago, when $\mathrm{Hu}$ and coworkers investigated the phylogenetic relationship between four toxic and three non-toxic strains. Their findings suggested a new species for insecticidal strains and provided evidence for toxicity evolution by means of horizontal gene transfer (HGT) [18]. However, a more comprehensive analysis is required as the number of available genome sequences has doubled. Therefore, we aimed to perform a broader evaluation of the intraspecific genetic diversity of $L$. sphaericus as species and as mosquito-control agent.

\section{Results}

A new $L$. sphaericus genome is now available

The genome of $L$. sphaericus OT4b.49, a previously isolated Colombian strain [14], was sequenced using Pacific Biosciences technology and assembled, obtaining 3 contigs (4.6 Mbp, $29.6 \mathrm{Kbp}$ and $14.9 \mathrm{Kbp}$ ) and no evidence of plasmids. The estimated coverage was $242 \times$ with 981,395,594 bp produced, of which 11,921,224 bp were from circular consensus sequencing (CCS). Moreover, the GC content (37.30\%), predicted genes (4486) and genome size $(4.7 \mathrm{Mbp})$ were expected according to the currently reported genomes. This newly available genome together with the other $13 \mathrm{~L}$. sphaericus genomes previously uploaded to the NCBI database, were used in the current study (Table 1).

\section{S rDNA homology cannot differentiate toxic from non-toxic isolates}

L. sphaericus is generally classified into five DNAhomology groups based on the similarity of $16 \mathrm{~S}$ rDNA sequences. These groups are also related by some phenotypic traits and molecular markers [19]. We reconstructed the $16 \mathrm{~S}$ rDNA phylogeny including the $14 \mathrm{~L}$. sphaericus strains herein analyzed and found the same clustering pattern reported in previous studies $[19,20]$ with high bootstrap support. All the toxic strains are grouped together in the same lineage with some nontoxic strains and, separately, the other non-toxic strains are distributed among the other groups (Fig. 1). Therefore,

Table 1 L. sphaericus genomes used in this study

\begin{tabular}{|c|c|c|c|c|c|c|}
\hline Strain & Toxicity $^{a}$ & Level & Genome size (bp) & Contigs & Accession no. & Reference \\
\hline C3-41 & High & Complete & $4,639,821$ & $2^{\mathrm{b}}$ & CP000817 & {$[48]$} \\
\hline 2362 & High & Complete & $4,692,801$ & 1 & СР015224 & [49] \\
\hline III(3)7 & High & Complete & $4,663,526$ & $2^{b}$ & СР014856 & [14] \\
\hline OT4b.25 & High & Complete & $4.665,575$ & $2^{b}$ & СР014643 & [50] \\
\hline OT4b.49 & High & Draft & $4,668,840$ & 3 & LWHI01000000 & This study \\
\hline CBAM5 & High & Draft & $5,156,460$ & 93 & AYKQ00000000 & [13] \\
\hline LP1-G & High & Draft & $4,542,839$ & $143^{b}$ & JPDL01000000 & {$[17,51]$} \\
\hline 2297 & Medium & Draft & $4,516,760$ & 278 & JPDJ01000000 & [18] \\
\hline SSII-1 & Low & Draft & $4,651,985$ & 138 & JPDK01000000 & [18] \\
\hline 1987 & Non-toxic & Draft & $4,906,630$ & 70 & JMMU01000000 & Not published \\
\hline OT4b.31 & Non-toxic & Draft & $4,856,302$ & 94 & AQPX00000000 & [52] \\
\hline B1-CDA & Non-toxic & Draft & $4,509,276$ & 84 & LJYY01000000 & [12] \\
\hline KCTC 3346 & Non-toxic & Draft & $4,560,870$ & 83 & AUOZ00000000 & [53] \\
\hline NRS 1693 & Non-toxic & Draft & $4,603,690$ & 546 & JPDM01000000 & [18] \\
\hline
\end{tabular}

${ }^{a}$ High: presence of $\operatorname{bin} A, B$ and $m t x 1,2,3$ or cry 48,49 ; Medium: Only binA, $B$ and $m t x 2$; Low: only $m t x 2$. This classification was previously established by Ge et al. by associating the presence of toxin genes with mosquitocidal activities of 35 L. sphaericus isolates [54]

${ }^{\mathrm{b}}$ At least one plasmid-associated contig 


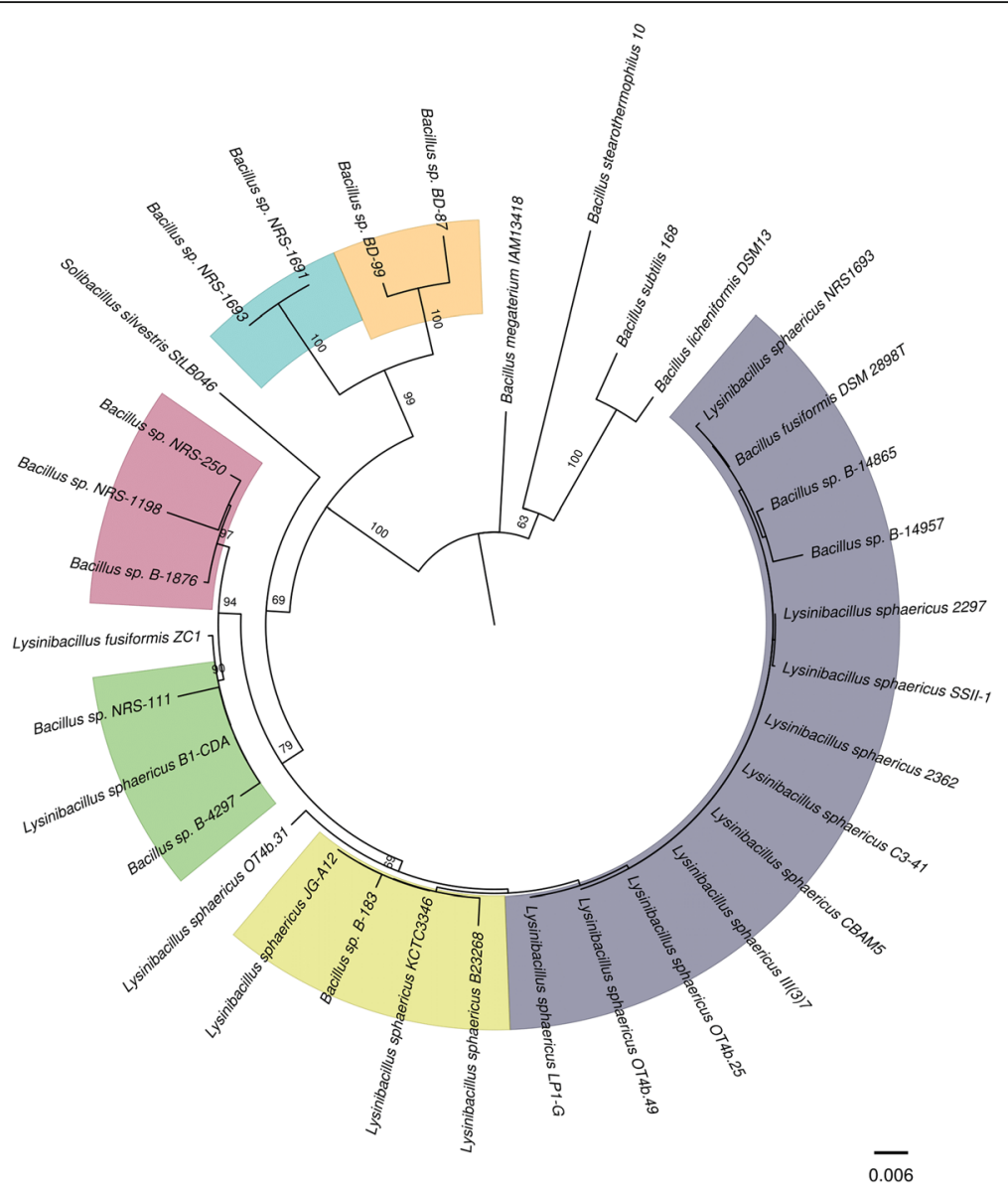

Fig. 1 Phylogenetic tree of round-spored bacilli showing the current 16S rDNA-based taxonomy. L. sphaericus strains can be found in three out of the six highlighted homology groups. All the toxic strains are clustered in the group in purple, however, not all the strains in that group are toxic. Bootstrap values for 500 replicates are shown in the branches

as it was suggested, only in the view of $16 \mathrm{~S} \mathrm{rDNA}$ homology, toxicity does not seem to be appropriate as the sole differentiator [21]. However, further analyses revealed high diversity within $L$. sphaericus and suggested that toxicity is a differentiating feature that could be acquired in a single evolutionary event.

\section{Toxic strains comprise a nearly clonal and independent lineage with a high degree of synteny}

We performed comparative genomic analyses which supported the hypothesis of the toxic strains as an independent group within $L$. sphaericus. The first evidence came from multiple genome alignments and the evaluation of genomic architectures carried out by Gepard [22] and MAUVE [23] softwares. The toxic strains exhibited strong syntenic relationships, though some rearrangements, mainly duplications, were detected (Fig. 2a). On the other hand, genomes from non-toxic strains showed several differences among them and in comparison to the toxic strains genomes, especially the strain B1-CDA, in which several inversions were identified (Fig. 2b).

An additional whole genome alignment, based on BLAST, was conducted and visualized using BRIG [24]. The ring image obtained clearly showed the high similarity between the toxic strains and a great heterogeneity when they are compared to the non-toxic ones (Fig. 3). Intriguingly, when this assay was performed with a toxic strain as a reference (as shown in Fig. 3), the divergence pattern among the non-toxic strains was very similar. This suggests the existence of gene clusters unique for the toxic strains beyond the toxin-encoding genes.

\section{HGT might have played a role in toxicity acquisition}

Eleven Genomic Islands (GIs) were detected for the strain OT4b.49. As reported by $\mathrm{Hu}$ and coworkers, the identified GIs comprise sequences of mobile genetic elements as prophages and transposons, and several recombination-involved proteins as integrase, recombinase, and transposase [18]. Interestingly, mosquitocidal toxin coding genes are within or in the immediacy of 

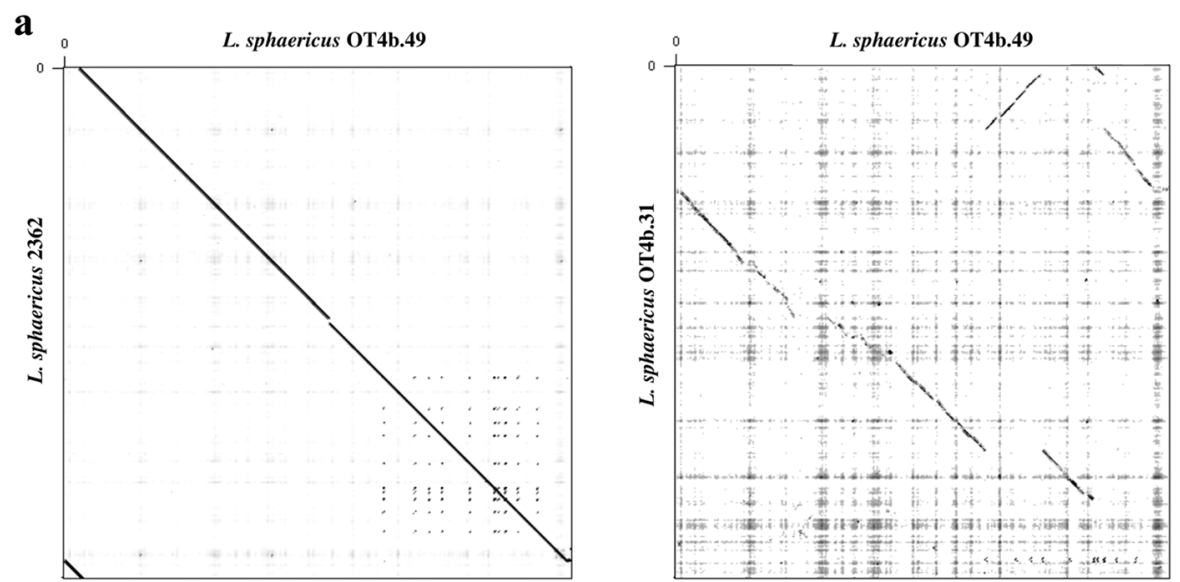

b

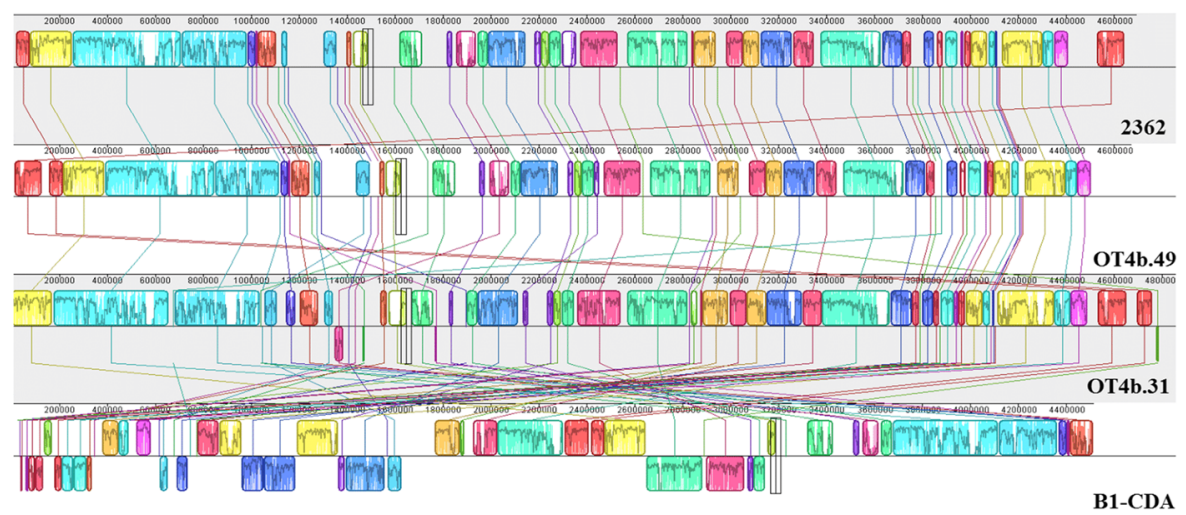

Fig. 2 Whole genome alignments between toxic and non-toxic strains. a Dot-plots of nucleotide identities of the toxic strains OT4b.49 against 2362 (left) and OT4b.49 against the non-toxic strain OT4b.31 (right). b Nucleotide-based alignment of the genomes from two toxic (OT4b.49 and 2362) and two non-toxic (OT4b.31 and B1-CDA) strains. Homologous blocks are shown as identically colored regions and linked across the genomes. Regions that are inverted relative to L. sphaericus 2362 are shown below the central axis of each sequence

those GIs (Fig. 3). All of the completed genomes from toxic strains were evaluated for GIs and, as it was previously hypothesized, all of them have between 7 and 11 GIs associated with the toxin genes. This suggests a role for these mobile large segments of DNA in the acquisition of entomopathogenic activity.

\section{The toxic lineage appears to represent a novel species}

Since some researchers have proposed that $L$. sphaericus could compromise of more than one species in a single sensu lato [18-20], we calculated the correlation indexes of tetranucleotide signatures (TETRA) and the Average Nucleotide Identity based on BLAST and MUMmer (ANIb and ANIm) as metrics to assess the species circumscription [25]. In the same way, we performed insilico genome-to-genome comparison to calculate digital DNA:DNA hybridization estimates (DDH) [26]. All the evaluated metrics allowed a clearer circumscription for the toxic lineage, providing support to the hypothesis of this group as a novel species. On the contrary, it is not clear if all the non-toxic strains belong to the same species or not because, although some identity values were below the threshold of species level, the results were not consistent across the metrics (Fig. 4). For instance, TETRA values indicated that the strains 1987, B1-CDA and NRS 1693 are right on the species boundary with the toxic group, in contrast to results from ANIb which suggested they are different species from each other. Besides the consensus for the toxic group, the three metrics designated the strains OT4b.31 and KCTC 3346 (L. sphaericus type strain) as the most divergent ones.

Furthermore, to gain a deeper insight on this matter, we aimed to reveal distinctive traits of toxic and nontoxic strain by identifying, evaluating, and comparing clusters of orthologous genes (COGs) from protein sequences comparisons. In concordance with results mentioned above, little functional diversity was found in 4 representatives of toxic lineage since no unique genes were detected for any strain (Additional file 1: Figure S1). However, when the same evaluation was carried out with two toxic and two non-toxic strains, a greater 


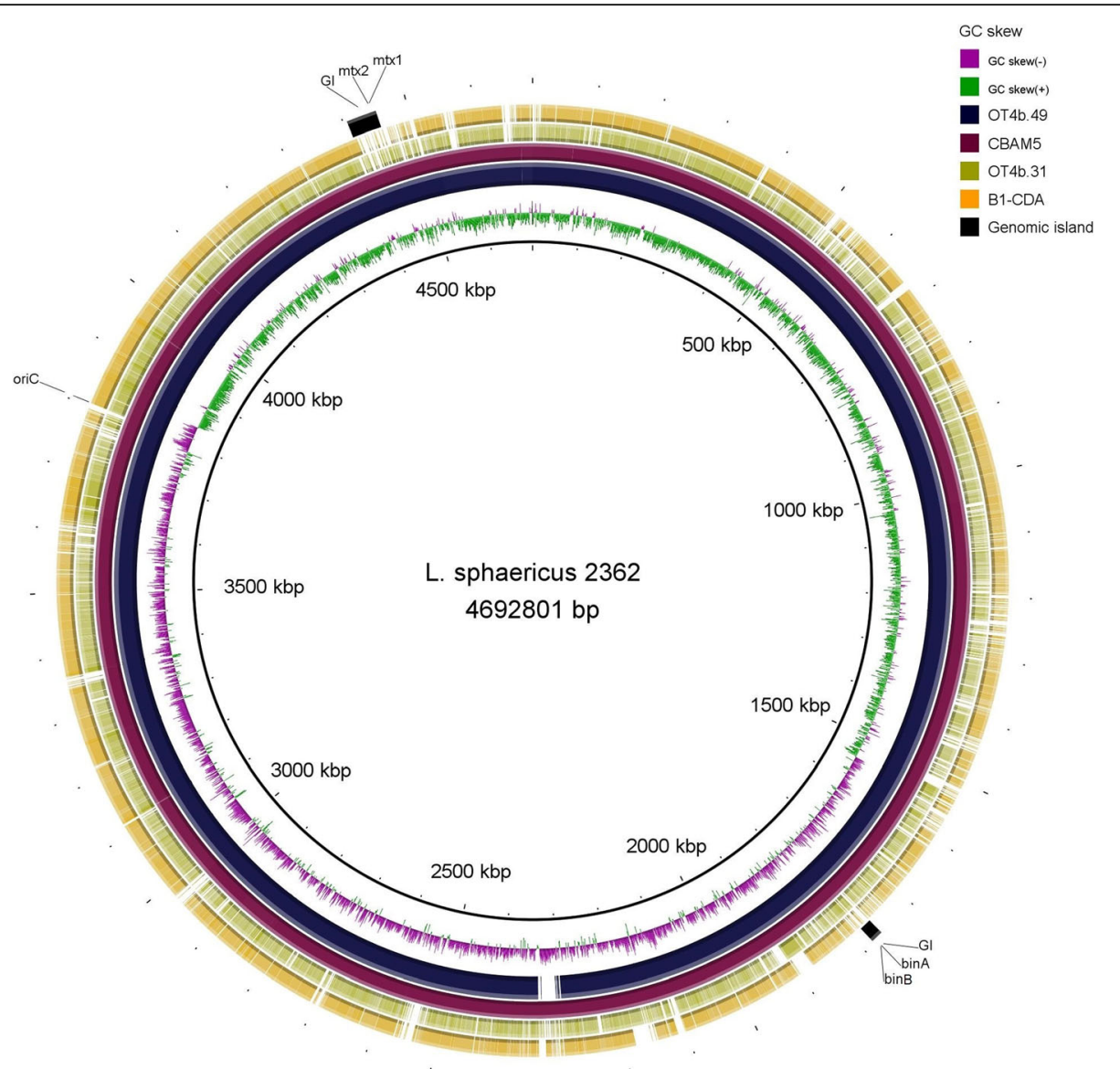

Fig. 3 Circular map that compares genomes of L. sphaericus OT4b.49, CBAM5, OT4b.31, and B1-CDA against 2362. Each circle represents the genome from one strain, and the colored blocks in it represent sequences with $>90 \%$ identity relative to L. sphaericus 2362 . The Gls in the immediacy of encoding toxin genes as well as the origin of replication are spotted. GC skew is shown in the inner circle

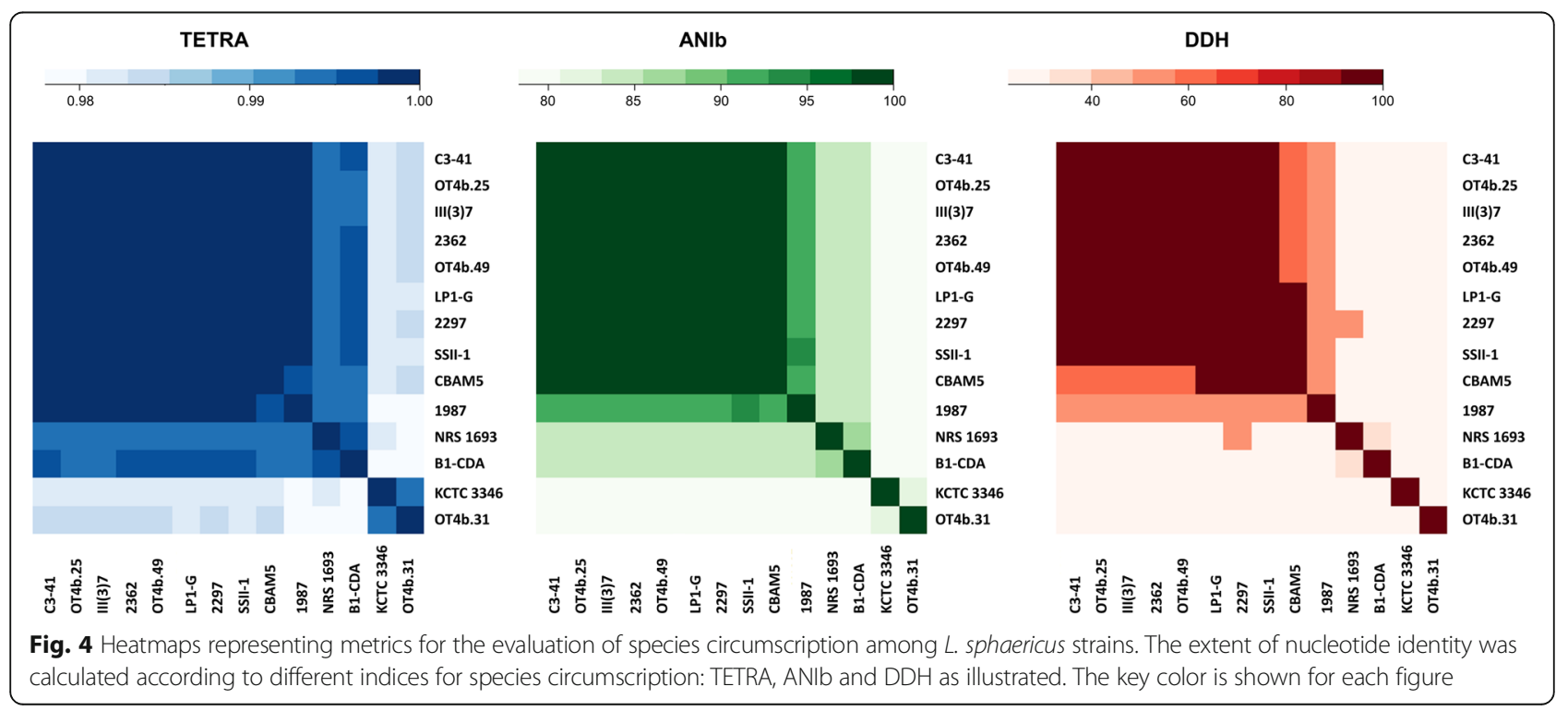


heterogeneity was observed, with the toxic strains being the ones which shared the highest amount of COGs (Additional file 1: Figure S1). The core and accessory genes of $L$. sphaericus as well as core and accessory genes of toxic lineage were identified by following Roary pipeline [27]. Three thousand seven hundred and twenty eight core genes (defined as genes in more than $95 \%$ of evaluated strains) were found in toxic lineage as well as a pangenome pool containing 5881 genes. In sharp contrast, only 391 genes constituted the core-genome and 20,217 genes constituted the pan-genome when both toxic and non-toxic strains were evaluated.

The phylogenetic tree constructed based on core genes supports the hypothesis about toxic strains as separate species from non-toxic strains, the latter without a clear circumscription (Fig. 5). Interestingly, the strain OT4b.31 was the most related with the strain KCTC 3346, which is the type strain for L. sphaericus [28]. Thus, this group would be $L$. sphaericus sensu stricto whereas toxic lineage should be considered as a new species.

Finally, we assessed the pan-genome of both the toxic lineage and $L$. sphaericus as it is described nowadays (comprising toxic and non-toxic strains). The pangenome of the toxic lineage of $L$. sphaericus was composed of 3728 core genes and 2153 accessory genes, whereas the pan-genome of the whole L. sphaericus species was larger, with 391 core genes and 19,826 accessory genes, which provides evidence for high intraspecies diversity (Fig. 6 and Additional file 2: Figure S2).

\section{Discussion}

It is commonly recognized that a few sequenced genomes may misrepresent the entire genetic repertoire of a species [29, 30]. That is why the current availability of $14 \mathrm{~L}$. sphaericus genomes has made this traditionally controversial group an excellent candidate for phylogenomics and pan-genomic studies that clarify the species boundaries for this taxon. In this work, we carried out a comprehensive analysis of $L$. sphaericus as a species and mosquito-control agent, obtaining results that suggest the need of a new species designation.

The results showed a high diversity within L. sphaericus, with entomopathogenicity being the main feature that allows a clear distinction among the strains. This supports previous studies whereby a reevaluation of $L$. sphaericus as a species was suggested. By convention, round-spored mesophilic bacilli that grow at neutral $\mathrm{pH}$ and are unable to ferment carbohydrates have been classified as L. sphaericus sensu lato [20]. As unique phenotypic traits are discovered, novel species have been designated from this group, this is the case for $L$. fusiformis, L. boronitolerans, and Sporosarcina globispora, among others [19]. As Nakamura states, the dependence of early studies on insensitive methods hindered estimation of diversity and fostered the creation of heterogeneous species that includes toxic and non-toxic strains [19]. Hence, variability in toxicity might arise from genetic variability and incorrect classification.

We found evidence that suggests the toxicity could have been acquired by a HGT event because toxin genes were found flanked by genomic islands containing several integrase, recombinase, and transposase sequences. However, we are still not able to clarify from what kind of gene transfer event the mosquitocidal activity arose.

It is very intriguing that all toxic strains shape a nearly clonal group in spite of their very different provenance:

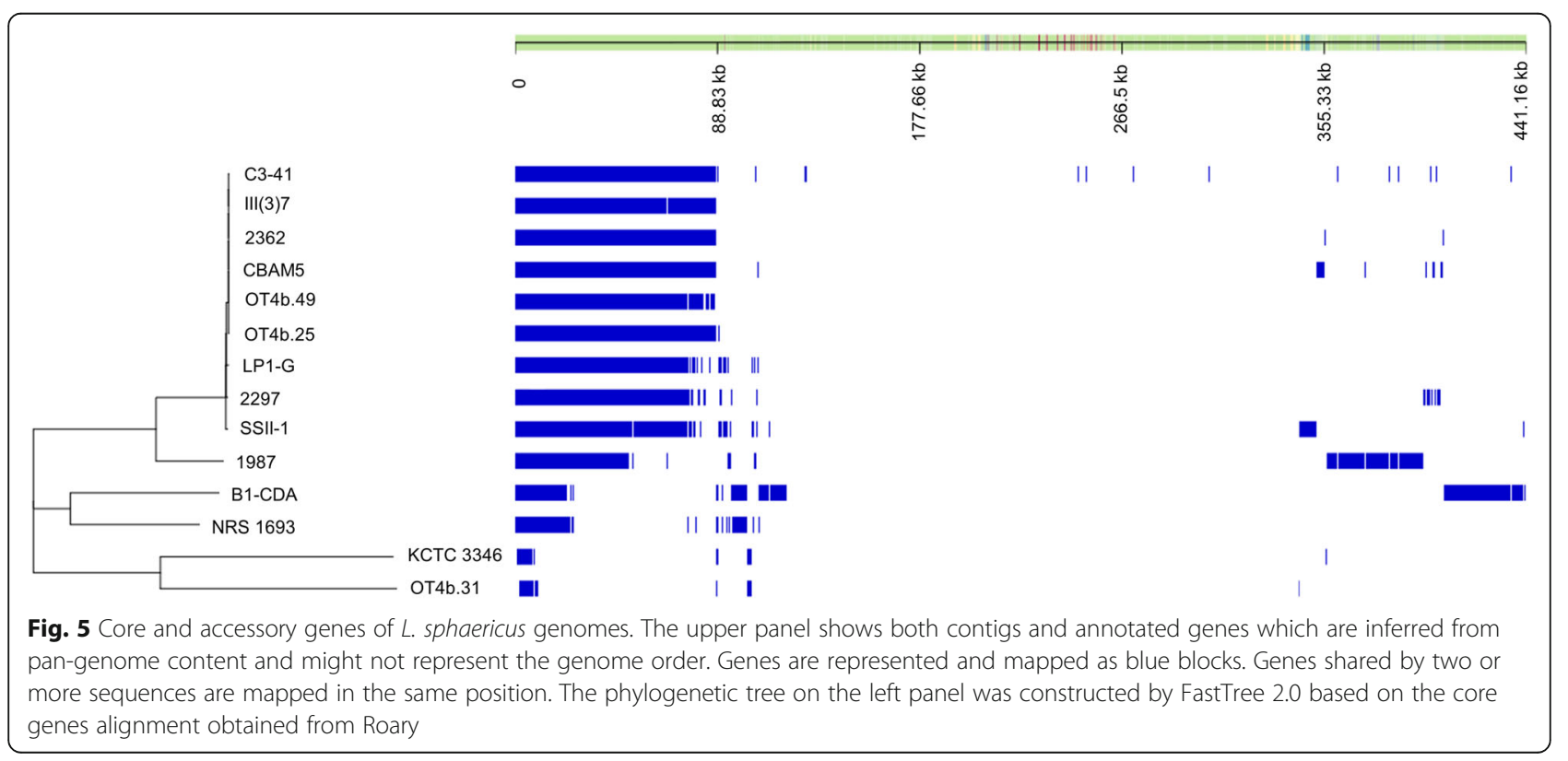


Toxic strains

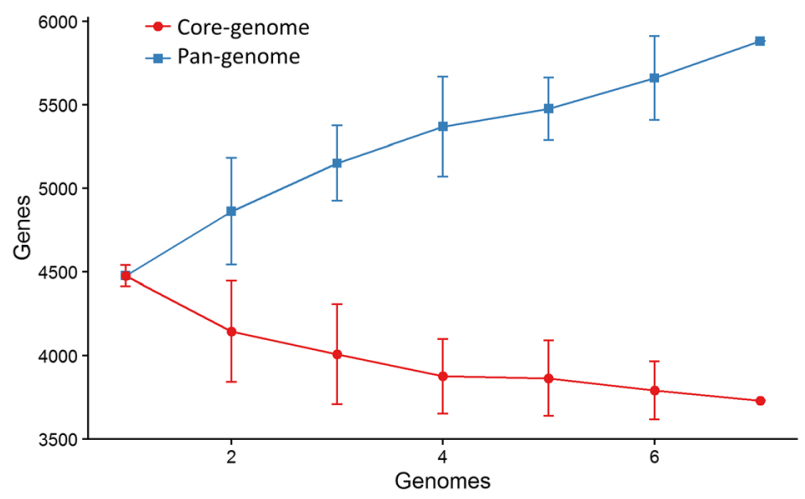

All strains

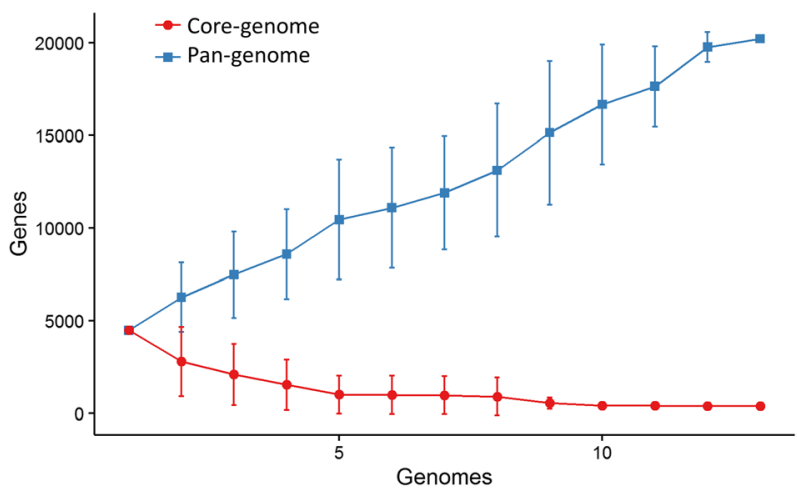

Fig. 6 Pan- and core-genome of L. sphaericus. The curves depict the pan and core-genome, for toxic strains and for the complete set of analyzed strains, both as function of the number of genomes

strains 2362, C3-41 and OT4b.49 were respectively isolated from Africa, Asia and South America. Therefore, we hypothesize that toxins acquisition lead to the emergence of the toxic lineage by providing a fitness increase and thus, a great genomic stabilization.

Since the toxic lineage are made up by nearly clonal strains, the sole presence of $\operatorname{bin} A$ or $\operatorname{bin} B$ (which are always together) and $m+x 2$ (or $c r y$ ) toxin-encoding genes is a good indicator of the feasibility for using a roundspored bacilli as mosquito-control agent. This could be easily assessed, for instance, by a PCR assay. In addition, these genes also would indicate the presence of other interesting core genes from this lineage, such as those that encode for S-layer protein and metal efflux pumps [14, 31].

Herein, we compared $14 \mathrm{~L}$. sphaericus genomes with one another by using ANIb, TETRA, and digital DDH, in order to achieve a clearer species circumscription $[25,26,32]$. The results certainly showed the toxic lineage of L. sphaericus as a single and independent species (Fig. 4 and Additional file 3: Table S1). A further evaluation of remaining members of the $L$. sphaericus species is required due to values outside the intra-species range ( $<96 \%$ and $<0.999$ for ANI and TETRA, respectively, and $<70 \%$ for DDH) in some non-toxic strains.

An alternative and novel way to describe a bacterial species is by its pan-genome, which is the sum of the core (genes present in all strains), dispensable (genes present in two or more strains), and unique (genes present in single strains) genomes [30]. As Tettelin and coworkers proposed, by defining the pan-genome of a bacterium, insights both on its biology and life style can be gained as well as implications for the definitions of the species itself [33]. Our comparative analysis of $14 \mathrm{~L}$. sphaericus genomes indicated a pan-genome with frequent rearrangements, revealing the striking genomic heterogeneity inside this group. When performing the same comparative analysis on the 8 entomopathogenic strains, an open but smaller pan-genome as well as highly syntenic regions and less frequent genomic rearrangements were found (Fig. 6).

Finally, it is important to take into account that frequent gaps and sequencing errors might cause underestimation in genome annotation and therefore, errors in the estimation of the core- and pan-genome [34]. Only 4 of the 14 genomes analyzed are completed as a single chromosomal contig, which constitutes an inherent limitation of this study and highlights the importance of technologies that make closed genomes possible.

\section{Conclusions}

We generated a draft genome for the Colombian mosquitocidal L. sphaericus OT4b.49 and carried out an analysis of the full repertoire of $L$. sphaericus available genomes in order to assess intraspecific diversity.

The current study provides strong evidence for considering the toxic-lineage of $L$. sphaericus as a new species. Historically, many round-spored mesophilic bacilli have been grouped under L. sphaericus classification, leading to the formation of a heterogeneous sensu lato. We assessed taxonomic composition by means of overall genome relatedness indices and phylogenomic analysis based on core genes. We found that toxic strains form a welldefined lineage that should be considered as a novel species. The differentiating feature of this species is the presence of toxin-encoding genes such as $\operatorname{bin} A, B$ and mtx 1, 2, 3, which might be acquired by HGT.

On the other hand, the remaining L. sphaericus strains did not show a clear circumscription and are, indeed, a paraphyletic group. Further studies are needed in order to establish a univocal classification, though this is still challenging in the light of the absence of an unambiguous species definition for bacteria. 


\section{Methods}

\section{Genome sequencing and assembly}

The genome sequencing of $L$. sphaericus OT4b.49 was carried out using Pacific Biosciences technology with 1 SMRT cell, P4-C2 chemistry, and a mixed library (CCS and subreads). This service was provided by McGill University and Génome Québec Innovation Centre. Contig assembly was done using the HGAP 2.0 workflow [35]. Sequencing errors were corrected by aligning multiple short reads on longer reads. Subsequently, the corrected reads were used as seeds into Celera Assembler [36] to obtain contigs. These contigs were polished through an alignment of raw reads on contigs by BLASR [37] and then, high quality consensus sequences were generated from these contigs by a variant calling algorithm (Quiver).

\section{Genome annotation}

The genome of L. sphaericus OT4b.49 was annotated using the NCBI Prokaryotic Genome Annotation Pipeline [38] and RAST [39]. In addition, the 14 genomes used in this study (Table 1) were re-annotated by Prokka [40], which locates ORFs by Prodigal and RNA regions using RNAmmer, Aragorn, SignalP and Infernal. Then, it annotates the translated sequences by a homology searching with BLAST and HMMER, followed by a searching against public databases (CDD, PFAM, and TIGRFAM) and the Prokka "Kingdom Bacteria" database.

\section{S rDNA phylogeny}

The 16S rDNA sequences obtained with Prokka, together with the 16S rDNA sequences from other bacilli listed below, were aligned using MEGA 6.0 [41] with the MUSCLE algorithm. The phylogenetic tree was then constructed by the neighbor-joining method and the distances, computed with the Kimura's two-parameter model [42] using only positions with $>95 \%$ coverage. Bootstrap tests were carried out with 500 replicates. The additional $16 \mathrm{~S}$ rDNA sequences were: Bacillus subtilis $168 \mathrm{~T}$ (X60646), Bacillus licheniformis DSM 13T (X68416), Bacillus megaterium IAM 13418T (D16273), Bacillus sp. BD-87 (AF169520), Bacillus sp. BD-99 (AF169525), Bacillus sp. NRS-1691 (AF169531), Bacillus sp. NRS-1693 (AF169533), Solibacillus silvestris StLB046 (NR_074954), Bacillus sp. NRS-250 (AF169536), Bacillus sp. B-1876 (AF169494), Bacillus sp. NRS-1198 (AF169528), Bacillus sp. B-4297 (AF169507), Bacillus sp. NRS-111 (AF169526), Bacillus sp. B-183 (AF169493), Lysinibacillus sphaericus B-23268T (AF169495), Lysinibacillus sphaericus JG-A12 (AM292655), Bacillus sp. B-14905 (AF169491), Lysinibacillus sphaericus ZC1 (NZ_ADJR01000054.1:1-1487), Bacillus sp. B-14865 (AF169490), Lysinibacillus fusiformis ATCC-7055 (AJ310083), Bacillus sp. B-14957 (AF169492) and Bacillus sp. B-23269 (AF169496).

\section{Genome comparison}

MAUVE software [23] was used in order to perform whole genome alignments and synteny comparisons. Genomes were also compared with BRIG [24] and the genomic islands and toxin-encoding genes, previously predicted, were mapped in this comparison. Dot plots were generated by Gepard [22] using the ordered contigs produced by MAUVE for each genome.

\section{Identification of genomic islands}

Genomic islands were predicted in the complete genomes of toxic L. sphaericus strains using Island Viewer 3 [43]. This tool integrates the IslandPick, IslandPath.DIMOB and SIGI-HMM algorithms.

\section{Average nucleotide identity, correlation indexes, and DDH estimates}

The values for ANIb, ANIm and Tetra were calculated by JSpecies [32] for all the possible strain pairs among L. sphaericus genomes. DDH estimates were obtained from Genome to Genome Distance Calculator 2.1, which transforms the distances from the high-scoring segment pairs to values analogous to DDH using a generalized linear model. This model is inferred from an empirical reference dataset comprising real DDH values and genome sequences [26]. All of the results above were represented as heatmaps using $R$ statistical software [44].

\section{Pan- and core-genome analysis}

The pan- and core-genomes for all strains of $L$. sphaericus as well as for the toxic and non-toxic strains were obtained using OrthoMCL [45] and Roary (with codon aware alignment) [27]. Roary uses FastTree 2.0 algorithm to infer an approximatelymaximum-likelihood tree from large alignments by the Jukes-Cantor model for nucleotide evolution [27]. The results from Roary were visualized by Phandango [46] as a phylogenetic tree of core genes and by $R$ statistical package as graphs of number of genes vs number of genomes. Orthologous gene clusters were identified and visualized by OrthoVenn which follows a similar pipeline to OrthoMCL [47].

\section{Additional files}

Additional file 1: Figure S1. Shared and unique COGs. The Venn diagrams indicate the number of shared and unique COGs across representative toxic, non-toxic and a mixed group of strains. (PNG $251 \mathrm{~kb}$ )

Additional file 2: Figure S2. New and unique genes in L. sphaericus pan-genome. The curves depict new and unique genes found with the addition of new genome sequences for toxic and for the complete set of analyzed strains. (PNG $140 \mathrm{~kb}$ ) 
Additional file 3: Table S1. TETRA, ANIb and DDH estimates. The matrices indicate the value for indices TETRA, ANIb, and ANIm, as well as DDH estimates for all the studied L. sphaericus strains. Intra species values are colored as green and interspecies values, as red. Orange color indicates the species boundary where further analyses are required. Numbers in brackets represent the nucleotides used as seed for the respective BLAST search. Threshold values were used as indicated by Rosselló-Móra and Amann [25]. (XLSX 17 kb)

\section{Acknowledgments}

We would like to thank Dr. Alejandro Reyes Muñoz for his helpful discussions and guidance during this work.

Additionally, we thank Tito David Peña-Montenegro and Elizabeth Dell Trippe (University of Georgia) for their kind reviewing of the manuscript and their invaluable suggestions.

\section{Funding}

This project was supported by Faculty of Sciences of Universidad de los Andes. The funding entity played no part in the design of the study, collection, analysis, and interpretation of the data or in writing the manuscript.

\section{Availability of data and materials}

The genome sequences used in the current study are available on the NCB Genome Database under the accession numbers listed in Table 1.

\section{Authors' contributions}

Conceived and designed the experiments: JD. Performed the experiments: CGG and AHS. Analyzed the data: CGG and AHS. Contributed reagents/ materials/analysis tools and acquired funding: JD. Wrote the paper: CGG, AHS and JD. All authors read and approved the final manuscript.

\section{Competing interests}

The authors declare that they have no competing interests.

\section{Consent for publication}

Not applicable.

\section{Ethics approval and consent to participate} Not applicable.

Received: 11 June 2016 Accepted: 27 August 2016 Published online: 05 September 2016

\section{References}

1. Berry C. The bacterium, Lysinibacillus sphaericus, as an insect pathogen. J Invertebr Pathol. 2012;109:1-10.

2. Broadwell AH, Baumann L, Baumann P. The 42- and 51-kilodalton mosquitocidal proteins of Bacillus sphaericus 2362: Construction of recombinants with enhanced expression and in vivo studies of processing and toxicity. J Bacteriol. 1990;172:2217-23.

3. Yousten AA, Davidson EW. Ultrastructural analysis of spores and parasporal crystals formed by Bacillus sphaericus 2297. Appl Environ Microbiol. 1982:44:1449-55.

4. Kellen WR, Clark TB, Lindegren JE, Ho BC, Rogoff MH, Singer S. Bacillus sphaericus Neide as a pathogen of mosquitoes. J Invertebr Pathol. 1965;7:442-8,

5. Silva Filha MHNL, Berry C, Regis L. Advances in Insect Physiology: Insects Midgut and Insecticidal Proteins. In: Dhadialla T, Gill S, editors. Advances in Insect Physiology. London: Elsevier; 2014. p. 89-176.

6. Myers P, Yousten AA. Toxic activity of Bacillus sphaericus SSII-1 for mosquito larvae. Infect Immun. 1978;19:1047-53.

7. Baumann L, Baumann P. Expression in Bacillus subtilis of the 51- and 42kilodalton mosquitocidal toxin genes of Bacillus sphaericus. Appl Environ Microbiol. 1989:55:252-3.

8. Baumann L, Broadwell AH, Baumann P. Sequence analysis of the mosquitocidal toxin genes encoding 51.4- and 41.9-kilodalton proteins from Bacillus sphaericus 2362 and 2297. J Bacteriol. 1988;170:2045-50.

9. Thanabalu T, Porter AG. A Bacillus sphaericus gene encoding a novel type of mosquitocidal toxin of $31.8 \mathrm{kDa}$. Gene. 1996;170:85-9.
10. Wirth MC, Berry C, Walton WE, Federici BA. Mtx toxins from Lysinibacillus sphaericus enhance mosquitocidal cry-toxin activity and suppress cryresistance in Culex quinquefasciatus. J Invertebr Pathol. 2014;115:62-7.

11. Russell BL, Jelley SA, Yousten AA. Carbohydrate metabolism in the mosquito pathogen Bacillus sphaericus 2362. Appl Environ Microbiol. 1989;55:294-7.

12. Rahman A, Nahar N, Nawani NN, Jass J, Ghosh S, Olsson B, et al. Comparative genome analysis of Lysinibacillus sphaericus B1-CDA, a bacterium that accumulates arsenics. Genomics. 2015;106:384-92.

13. Peña-Montenegro T, Lozano L, Dussán J. Genome sequence and description of the mosquitocidal and heavy metal tolerant strain Lysinibacillus sphaericus CBAM5. Stand Genomic Sci. 2015;10:1-10.

14. Lozano LC, Dussán J. Metal tolerance and larvicidal activity of Lysinibacillus sphaericus. World J Microbiol Biotechnol. 2013;29:1383-9.

15. He H, Qian TT, Liu WJ, Jiang H, Yu HQ. Biological and chemical phosphorus solubilization from pyrolytical biochar in aqueous solution. Chemosphere. 2014;113:175-81.

16. He H, Yuan SJ, Tong ZH, Huang YX, Lin ZQ, Yu HQ. Characterization of a new electrochemically active bacterium, Lysinibacillus sphaericus D-8, isolated with a $\mathrm{WO}_{3}$ nanocluster probe. Process Biochem. 2014:49:290-4.

17. Ahmed I, Yokota A, Yamazoe A, Fujiwara T. Proposal of Lysinibacillus boronitolerans gen. nov. sp. nov., and transfer of Bacillus fusiformis to Lysinibacillus fusiformis comb. nov. and Bacillus sphaericus to Lysinibacillus sphaericus comb. nov. Int J Syst Evol Microbiol. 2007;57:1117-25.

18. Xu K, Yuan Z, Rayner S, Hu X. Genome comparison provides molecular insights into the phylogeny of the reassigned new genus Lysinibacillus. BMC Genomics. 2015;16:140.

19. Nakamura LK. Phylogeny of Bacillus sphaericus- like organisms. Int J Syst Evol Microbiol. 2000;50:1715-22.

20. Alexander B, Priest FG. Numerical classification and identification of Bacillus sphaericus including some strains pathogenic for mosquito larvae. J Gen Microbiol. 1990;136:367-76.

21. Rippere KE, Johnson JL, Yousten AA. DNA similarities among mosquitopathogenic and nonpathogenic strains of Bacillus sphaericus. Int I Syst Bacteriol. 1997;47:214-6.

22. Krumsiek J, Arnold R, Rattei T. Gepard: a rapid and sensitive tool for creating dotplots on genome scale. Bioinformatics. 2007;23:1026-8.

23. Darling ACE, Mau B, Blattner FR, Perna NT. Mauve: multiple alignment of conserved genomic sequence with rearrangements. Genome Res. 2004;14:1394-403.

24. Alikhan N-F, Petty NK, Ben Zakour NL, Beatson SA. BLAST Ring Image Generator (BRIG): simple prokaryote genome comparisons. BMC Genomics. 2011;12:402.

25. Rossello-Mora R, Amann R. Past and future species definitions for Bacteria and Archaea. Syst Appl Microbiol. 2015;38:209-16.

26. Auch AF, von Jan M, Klenk H-P, Göker M. Digital DNA-DNA hybridization for microbial species delineation by means of genome-to-genome sequence comparison. Stand Genomic Sci. 2010;2:117-34.

27. Page AJ, Cummins CA, Hunt M, Wong VK, Reuter S, Holden MTG, et al. Roary: rapid large-scale prokaryote pan genome analysis. Bioinformatics. 2015;31:3691-3.

28. Baumann P, Clark MA, Baumann L, Broadwell AH. Bacillus sphaericus as a mosquito pathogen: properties of the organism and its toxins. Microbiol Rev. 1991;55:425-36.

29. Deng X, Phillippy AM, Li Z, Salzberg SL, Zhang W. Probing the pan-genome of Listeria monocytogenes: new insights into intraspecific niche expansion and genomic diversification. BMC Genomics. 2010;11:500.

30. Medini D, Donati C, Tettelin H, Masignani V, Rappuoli R. The microbial pan-genome. Curr Opin Genet Dev. 2005;15:589-94.

31. Pollmann K, Raff J, Schnorpfeil M, Radeva G, Selenska-Pobell S. Novel surface layer protein genes in Bacillus sphaericus associated with unusual insertion elements. Microbiology. 2005;151:2961-73.

32. Richter M, Rosselló-Móra R. Shifting the genomic gold standard for the prokaryotic species definition. Proc Natl Acad Sci. 2009:106:19126-31.

33. Tettelin H, Riley D, Cattuto C, Medini D. Comparative genomics: the bacterial pan-genome. Curr Opin Microbiol. 2008:11:472-7.

34. Roberts RJ, Carneiro MO, Schatz MC. The advantages of SMRT sequencing Genome Biol. 2009:14:133-8.

35. Chin C-S, Alexander DH, Marks P, Klammer AA, Drake J, Heiner C, et al. Nonhybrid, finished microbial genome assemblies from long-read SMRT sequencing data. Nat Methods. 2013;10:563-9. 
36. Myers EW. A Whole-Genome Assembly of Drosophila. Science 2000;287:2196-204.

37. Chaisson MJ, Tesler G. Mapping single molecule sequencing reads using basic local alignment with successive refinement (BLASR): application and theory. BMC Bioinformatics. 2012;13:238.

38. Angiuoli SV, Gussman A, Klimke W, Cochrane G, Field D, Garrity G, et al. Toward an online repository of Standard Operating Procedures (SOPs) for (meta)genomic annotation. OMICS. 2008;12:137-41.

39. Overbeek R, Olson R, Pusch GD, Olsen GJ, Davis JJ, Disz T, et al. The SEED and the Rapid Annotation of microbial genomes using Subsystems Technology (RAST). Nucleic Acids Res. 2014;42:D206-14.

40. Seemann T. Prokka: rapid prokaryotic genome annotation. Bioinformatics. 2014:30:2068-9.

41. Tamura K, Stecher G, Peterson D, Filipski A, Kumar S. MEGA6: Molecular Evolutionary Genetics Analysis version 6.0. Mol Biol Evol. 2013:30:2725-9.

42. Kimura M. A simple method for estimating evolutionary rates of base substitutions through comparative studies of nucleotide sequences. J Mol Evol. 1980;16:111-20.

43. Dhillon BK, Laird MR, Shay JA, Winsor GL, Lo R, Nizam F, et al. IslandViewer 3: more flexible, interactive genomic island discovery, visualization and analysis. Nucleic Acids Res. 2015;43:W104-8.

44. R Development Core Team. R: A language and environment for statistical computing. R Foundation for Statistical Computing, editor. Vienna, Austria; 2008.

45. Li L, Stoeckert CJ, Roos DS. OrthoMCL: identification of ortholog groups for eukaryotic genomes. Genome Res. 2003:13:2178-89.

46. Hadfield J, Harris S. Phandango. Wellcome Trust Sanger Inst. 2016. http://jameshadfield.github.io/phandango/. Accessed 25 May 2016.

47. Wang Y, Coleman-Derr D, Chen G, Gu YQ. OrthoVenn: a web server for genome wide comparison and annotation of orthologous clusters across multiple species. Nucleic Acids Res. 2015;43:W78-84.

48. Hu X, Fan W, Han B, Liu H, Zheng D, Li Q, et al. Complete genome sequence of the mosquitocidal bacterium Bacillus sphaericus C3-41 and comparison with those of closely related Bacillus species. J Bacteriol. 2008;190:2892-902.

49. Hernández-Santana A, Gómez-Garzón C, Dussán J. Complete Genome Sequence of Lysinibacillus sphaericus WHO Reference Strain 2362. Genome Ann. 2016;4:e00545-16.

50. Rey A, Silva-Quintero L, Dussán J. Complete Genome Sequence of the Larvicidal Bacterium Lysinibacillus sphaericus OT4b.25. Genome Ann. 2016;::e0257-16.

51. Wu E, Jun L, Yuan Y, Yan J, Berry C, Yuan Z. Characterization of a cryptic plasmid from Bacillus sphaericus strain LP1-G. Plasmid. 2007;57:296-305.

52. Peña-Montenegro TD, Dussán J. Genome sequence and description of the heavy metal tolerant bacterium Lysinibacillus sphaericus strain OT4b.31. Stand. Genomic Sci. 2013;9:42-56.

53. Jeong $H$, Jeong $D$, Sim Y. Genome sequence of Lysinibacillus sphaericus strain KCTC 3346. Genome Ann. 2013;1:e00625-13.

54. Ge Y, Hu X, Zheng D, Wu Y, Yuan Z. Allelic diversity and population structure of Bacillus sphaericus as revealed by multilocus sequence typing. Appl Environ Microbiol. 2011;77:5553-6.

\section{Submit your next manuscript to BioMed Central and we will help you at every step:}

- We accept pre-submission inquiries

- Our selector tool helps you to find the most relevant journal

- We provide round the clock customer support

- Convenient online submission

- Thorough peer review

- Inclusion in PubMed and all major indexing services

- Maximum visibility for your research

Submit your manuscript at www.biomedcentral.com/submit

Biomed Central 